This manuscript is a preprint and has not been submitted for traditional publication yet, we have a few options in mind but have not formally submitted a manuscript. Please note that this article has not undergone peer-review and the manuscript has yet to be formally accepted for publication. Subsequent versions of this manuscript may have slightly different content. If accepted, the final version of this manuscript will be available via the 'Peer-reviewed Publication DOI' link on the right-hand side of this webpage. Please feel free to contact any of the authors; we welcome feedback.

Example Citation: Mr. Jia D., Ridge High School, NJ USA, Dam Break Simulation with HEC-RAS and OpenFOAM. 2021.

Email: darrenjia2004@gmail.com 


\title{
Dam Break Simulation with HEC-RAS and OpenFOAM
}

\author{
Darren $\mathrm{Jia}^{1}$
}

September 21, 2021

\begin{abstract}
A dam break is a natural disaster that can cause significant property damage and loss of life. It's useful to identify potential flooding areas downstream in the event of a dam break. In this study both HEC-RAS [1] 2] and OpenFOAM 3] 4] are set up to simulate the inundation map downstream of the Dworshak dam in Idaho. Using the same topographical data from satellite observations, similar computational meshes are set up in both HEC-RAS and OpenFOAM. Where possible, identical or similar conditions are set up in HEC-RAS and OpenFOAM to model flooding patterns due to a dam break. The velocity of the water before reaching Ahsahka, the town located at the junction downstream from the dam, is $11.5 \%$ slower in HEC-RAS compared to OpenFOAM. The average velocity of water before reaching the end of the computational domain at Big Canyon Creek is about $20 \%$ slower in HEC-RAS compared to OpenFOAM. One notable discovery is that the water flow velocity in OpenFOAM appears to depend on the mesh resolution used in the simulation. A significant velocity difference is observed when water flows from one mesh refinement region to another mesh refinement region with a different resolution.
\end{abstract}

1

\section{Introduction}

Recent dam breaks in Brumadinho Brazil [8 [5, the iron range of Minnesota [6], and the state of Uttarakhand in India [7] remind us of the potential dangers of flooding due to dam breaks. HEC-RAS together with GIS [9] and satellite observations can be used to model such events. OpenFOAM provides an alternative approach to model such an event by performing a full 3 dimensional fluid dynamics simulation. In this study we apply both computational fluid

\footnotetext{
${ }^{1}$ darrenjia@bernardsboe.com, Ridge High School, NJ $U S A$
}

dynamics (CFD) softwares to examine the potential flooding that could occur from a hypothetical dam break at Dworshak Dam.

Dworshak Dam (Figure 11) is a concrete gravity dam in the United States, on the North Fork Clearwater River in Clearwater County, Idaho. With a height of $219 \mathrm{~m}$, Dworshak is the third tallest dam in the United States and the tallest straight-axis concrete dam in the Western Hemisphere. On average the dam has a volumetric discharge rate about $169.9 \mathrm{~m}^{3} / \mathrm{s}$. There are three downstream river stations monitoring water discharge (river station ids: 13340000, 13341550, 13341040) managed by USGS national water resources. The nominal water flow velocity downstream of the dam in Clearwater river is estimated to be less than $10 \mathrm{~m} / \mathrm{s}$ on average using 
the river station data.

While the dam is currently intact, it's interesting to compare OpenFOAM with HEC-RAS for a hypothetical dam break simulation and evaluate the results from the solvers and the potential flooding that may occur downstream in an event where the dam breaks.

The digital elevation data is publicly available from USGS [10] at 1/3 arc second (a resolution around of 10 meters). This digital elevation data is used to generate a topography mesh in both HEC-RAS and OpenFOAM.

\section{Simulation Setup}

\subsection{OpenFOAM Setup}

OpenFOAM is a popular open source CFD package that supports a finite volume [3] approach to general computational fluid dynamics simulation. In addition, the finite volume of the mesh 13 is adaptive and can be refined near areas of interest to capture the details of the water flow near the rivers and populated areas.

To set up the dam break simulation, we first create a geometry of Dworshak Dam and the surrounding terrain using Blender (an open source CAD software, [14) and the elevation data from USGS. Then a finite volume mesh with a refinement zone following the downstream terrain is created to be used in the OpenFOAM solver.

\subsubsection{Dam Geometry}

The digital elevation data of the dam and the terrain is converted into a geometry (Figure 2) through Blender. The reservoir portion of the geometry is lowered to simulate the depth of the lake. This allows for water to be placed inside the reservoir in OpenFOAM. Note that the flat geometry above the reservoir is artificially placed so that there will be a sufficient amount of water for the simulation. We only use a small portion of the digital elevation map (compare with Figure 1) near Dworshak reservoir in the simulation. The actual body of water above the dam is roughly $4,277,708,640 \mathrm{~m}^{3}$ (by courtesy of USACE Dworshak Dam Reservoir data sheet) in volume. The artificial geometry allows more water to be stored and discharged in the OpenFOAM simulation. A trapezoidal breach (Figure 3 with a width of $140 \mathrm{~m}$ at the top, width of 60 meters at the bottom, and height of 30 meters is artificially created in the center of the dam.

\subsubsection{Meshing}

To utilize the $1 / 3$ arc second resolution from the digital elevation map, different levels of mesh refinement are applied to create a mesh that matches the terrain for the simulation (Figure 4). A refined mesh in the shape of a box that surrounds the reservoir and the dam can be seen in Figure 4 near the right border. Rivers and areas of high population are further refined with visibly higher resolutions. Finally, the dam breach and portion of river immediately downstream from the dam are given the greatest refinement. As discussed later in Section 4.3, the resolution difference between different refinement regions causes a change in water velocity in OpenFOAM's simulation.

The coarse resolution mesh covering the entire computational domain has a cell resolution of $232.04 \times 211.23 \times 55.5$ in meters. To better capture the flooding pattern, areas of interest, e.g. rivers and potential flooding regions have increased resolutions with higher mesh refinement levels. Each refinement level increases the coarse mesh resolution by a factor of 2 . The area 

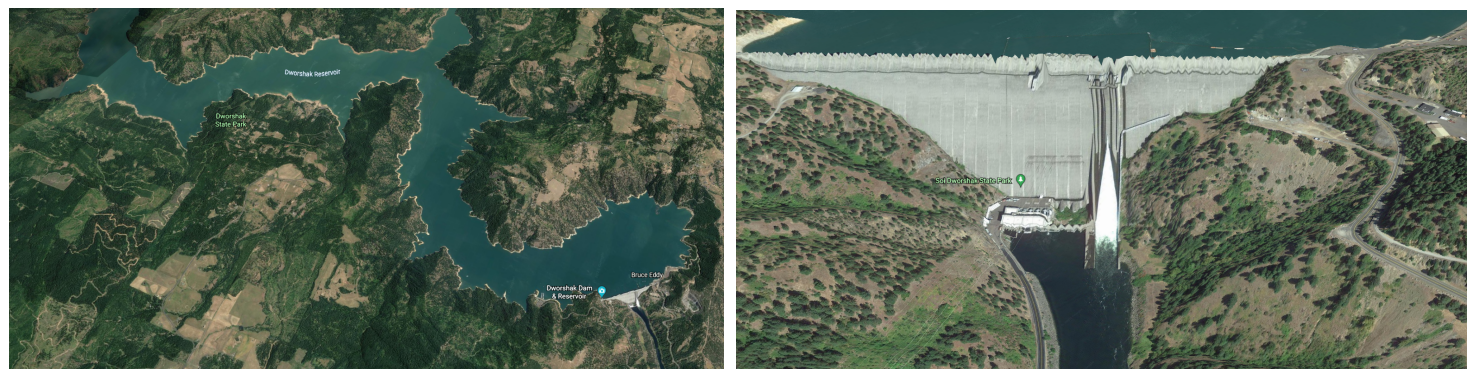

Figure 1: Dworshak Reservoir, Idaho (By courtesy of google Earth).

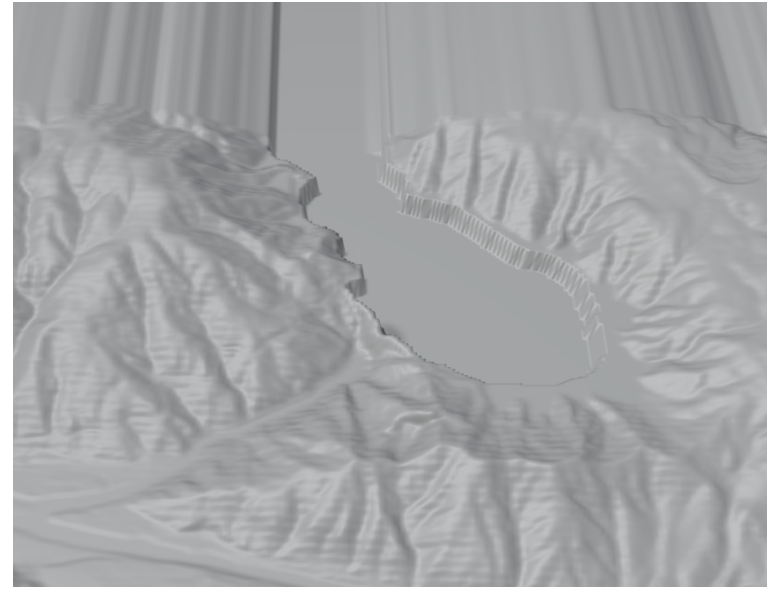

Figure 2: Geometry near the Dworshak reservoir imported in OpenFOAM

surrounding the reservoir with water to be discharged has a resolution of $116.02 \times 105.62 \times 27.8$ meters. Areas with high population and the portions of river far from the dam break have a resolution of $58.01 \times 52.81 \times 13.9$ meters. The dam breach and the portion of river immediately downstream from the dam have a resolution of $29.01 \times 26.40 \times 6.9$ meters. The entire OpenFOAM mesh has 198139 cells in total. The resulting mesh is shown in Figure 4 where the outlines of the downstream river and flooding regions are clearly visible from the cell resolution

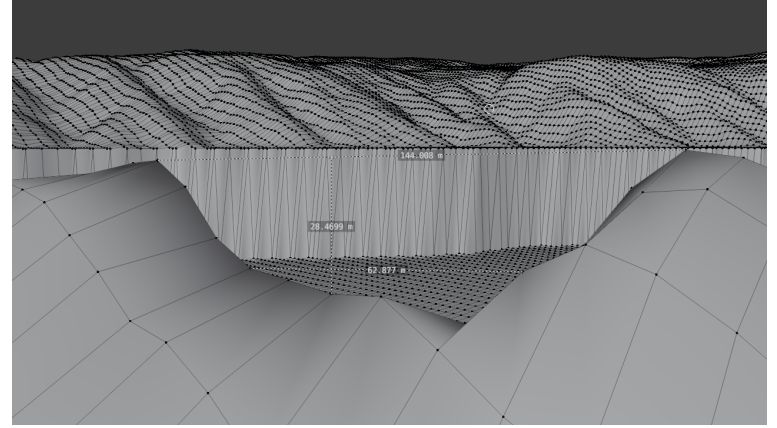

Figure 3: Geometry of the Dam: The top has a width of around $140 \mathrm{~m}$, the bottom has a width of around 60 meters and the height is around 30 meters. Due to the $10 \mathrm{~m}$ resolution of the TIF file, the curved shape of the dam, along with the inaccuracies associated with meshing in OpenFOAM, it is difficult to get more precise measurements of the size of the breach unlike in HEC-RAS which can specify the breach geometry precisely.

\section{contrast.}

To model water flow on an open terrain, OpenFOAM's Inter solver is used. The Inter solver works with 2 different phases of fluids: water and air. The shape of the reservoir is approximated using 3 boxes and a cylinder as shown in figure 5. Cells within the geometries are then 
set to water whereas all other cells are filled with air.

The fluid in the event of a dam break would likely be muddy as opposed to pure water. To simulate the muddy conditions of the water the parameters for activated sludge [16] [17] [18] [19] are used along with the Casson transport model [22] 23] instead of the default Newtonian model, allowing us to give the water a time dependent viscosity. The water is given a density of $1000 \mathrm{~kg} / \mathrm{m}^{3}$ which is identical to what's used in HEC-RAS.

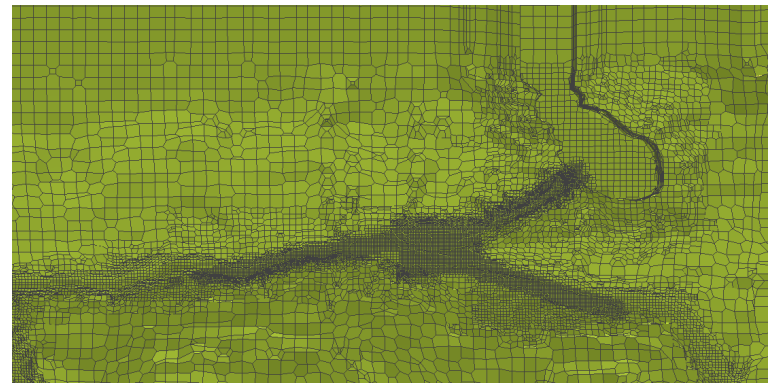

Figure 4: Meshing of the terrain: Areas of high interest such as rivers, high population areas, and the dam breach have visibly higher levels of refinement and better resolutions.

\subsection{HEC-RAS Setup}

HEC-RAS is developed by the US Army Corps of Engineers (USACE) for hydrology modelling. One of the strengths of HEC-RAS is flooding simulations associated with dam breaks. Setting up the GIS projection of the digital elevation data, meshing, and configuring the solver are streamlined in HEC-RAS, making the simulation setup much easier.

A 2D storage area (SA) is created following the shape of the reservoir (Figure 6). The 2D

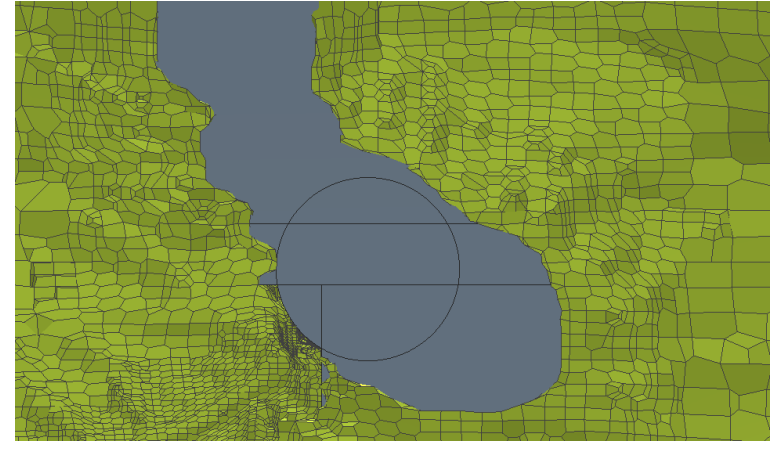

Figure 5: Parts of the mesh are specified as water through the use of 3 boxes and a cylinder. The geometries fill the reservoir completely and match the height of the water level in HEC-RAS.

storage area is used to store water to be discharged and is connected to a downstream flow area through the dam. A $2 \mathrm{D}$ mesh is created downstream from the dam with a resolution of $30 \times 30$ meters which matches the resolution of the finest resolution cells in OpenFOAM. An SA connection breach (Figure 7) is created between the storage area and the $2 \mathrm{D}$ mesh in order to allow for water to flow between the 2 areas. This SA connection breach is then breached with a trapezoidal dam break with a width of 140 meters and 60 meters on the top and bottom respectively and a height of 30 meters. The dam forms in 0 seconds in order to mimic the setup in OpenFOAM since there is presently no way to specify a breach formation time in OpenFOAM such that the breach can develop gradually. The 2D mesh (Figure 8) follows the shape of the river with outlets placed once the river exceeds the bounds meshed in OpenFOAM. The simulation uses default HEC-RAS settings with the exception of using the SWE-EM (stricter momentum) equation instead of Diffusion Wave and a Manning's number of 0.03 , which is within the range 
of Manning's number for firm soil [21].

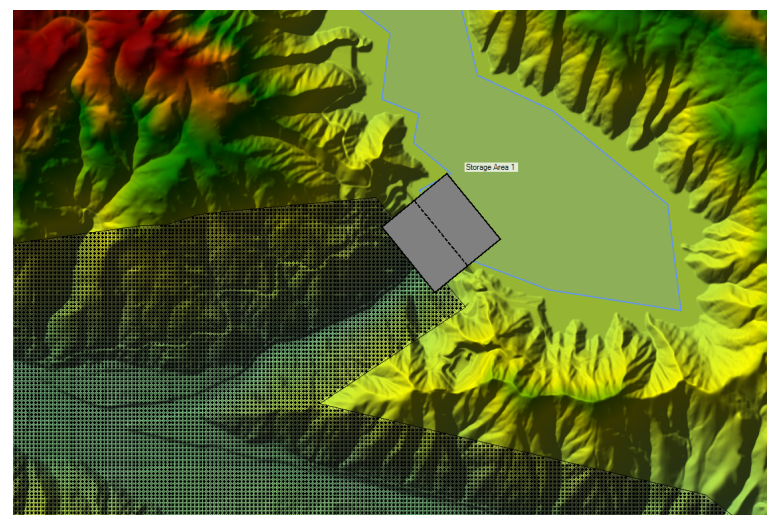

Figure 6: 2D storage area connected to downstream mesh through SA connection breach in HEC-RAS

\section{Dam Breach Shape}

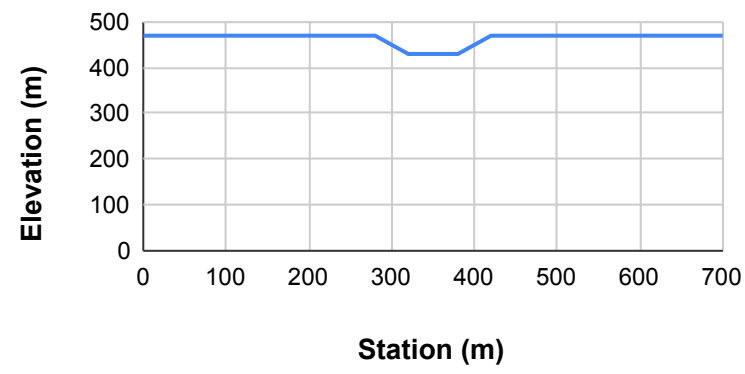

Figure 7: Breach in HEC-RAS

\subsection{Boundary Condition}

The bottom boundary that follows the shape of the terrain is set to a rough wall. In HEC-RAS, A Manning's number of 0.03 is used to simulate wall roughness for the terrain. In OpenFOAM the wall roughness boundary condition is set to a nutURoughWallFunction, giving friction to the

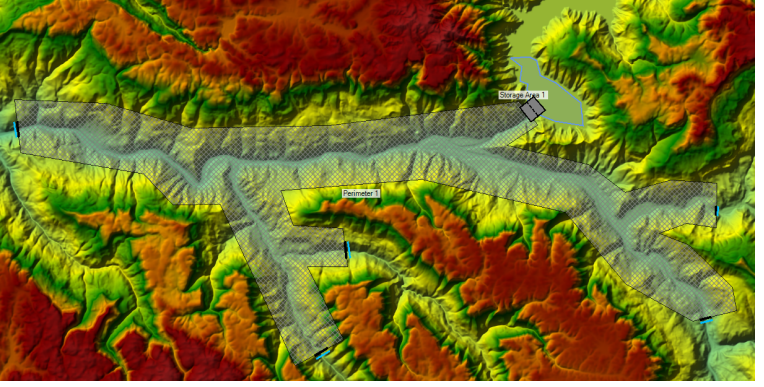

Figure 8: HEC-RAS is setup such that the mesh covers the river and mirrors the terrain in OpenFOAM

terrain. We have chosen a roughness height of $K_{s}=0.05$, a roughness constant of $C_{s}=1$, and a roughness factor 100 . The rough wall can also be set to a nutkRoughWallFunction which is not exercised in this study. Examining the documentation and source code of OpenFOAM, it's found that the roughness factor is applied to the roughness height in the boundary layer friction calculation (Equation 1 by courtesy of OpenFOAM documentation and source code). Having a roughness factor of 100 is equivalent to multiplying the roughness height by 100 .

$$
\begin{aligned}
R e & =\frac{u_{p} y}{\nu_{w}}+\zeta \\
\frac{d K s^{+}}{d y^{+}} & =\frac{\text { roughness factor } * \text { roughness height }}{y} \\
\nu_{t} & =0 \quad \text { if } y^{+}<=y_{\text {lam }}^{+} \\
\nu_{t} & =\nu_{w}\left(\frac{\left(y^{+}\right)^{2}}{R e}-1\right) \quad \text { if } y^{+}>y_{\text {lam }}^{+}
\end{aligned}
$$

No transformation exists currently from Manning's number $n$ used in HEC-RAS to roughness parameters used in OpenFOAM. The final roughness wall boundary condition for the terrain is chosen such that OpenFOAM most 
closely matches the distance traveled by the water modelled in HEC-RAS in the same amount of time.

\subsection{Initial Condition}

In order to match the simulation from HEC-RAS and OpenFOAM, both simulations start when the breach is fully formed. In HEC-RAS, this means the breach formation time is reduced to 0 . Then the extension of water flow and the flooding inundation map are compared between HEC-RAS and OpenFOAM.

\section{Solvers}

OpenFOAM's solver is a full $3 \mathrm{D}$ solver for the transient incompressible Navier-Stokes Equations. The thermodynamics aspects of the fluid flow are not modelled by the OpenFOAM solver chosen. HEC-RAS's solver is based on the 2D shallow water equations which does not simulate the thermodynamics aspects either. Both solvers allow for a dynamic time stepping constraint by prescribed Courant numbers. This feature allows the solvers to choose the largest time step possible that makes working with the variable resolution mesh possible and efficient throughout the simulation. Both simulations are calculation demanding, which means fixed time stepping can be extremely inefficient if the fixed time steps are set to values too small.

\subsubsection{OpenFOAM Solver}

The incompressible fluid solver in OpenFOAM uses a finite volume [3] approach to solve the incompressible viscous fluid flow [11] [12]. The governing equations of the incompressible vis- cous fluids are the typical incompressible steady Navier-Stokes equations:

$$
\begin{array}{r}
\nabla \vec{v}=0 \\
\frac{\partial \vec{v}}{\partial t}+\nabla \cdot(\vec{v} \otimes \vec{v})=-\nabla p+\nabla(\nu \nabla \vec{v})
\end{array}
$$

During each time step, the pressure-velocity coupled equation is solved by decoupling the pressure and momentum fields through predictor-corrector steps. During the momentum predictor step, $H$ matrix is solved from the momentum equation on the finite volume mesh.

$$
\begin{array}{r}
\nabla \cdot(\vec{v} \otimes \vec{v})-\nabla(\nu \nabla \vec{v})=-\nabla p \\
M V=-\nabla P \\
A=\operatorname{diag}(M) \\
H=A V-M V \\
A V-H=-\nabla P
\end{array}
$$

Now we can start the iterative process solving for pressure $P$ and velocity $V$. Start with the momentum equation,

$$
V=A^{-1} H-A^{-1} \nabla P
$$

Substituting the $V$ equation into continuity equation leads to a Poisson equation of pressure that can be solved by under relaxation method.

$$
\begin{array}{r}
\nabla V=\nabla\left(A^{-1} H-A^{-1} \nabla P\right)=0 \\
\nabla\left(A^{-1} \nabla P\right)=\nabla\left(A^{-1} H\right)
\end{array}
$$

In the predictor step, the momentum equation is solved using initial pressure $P$ and velocity boundary condition to find velocity $V$

$$
M V=-\nabla P
$$


The velocity field $V$ is then used in the Poisson equation to solve pressure $P$

$$
\nabla\left(A^{-1} \nabla P\right)=\nabla\left(A^{-1} H\right)
$$

The pressure field is then used in equation ( 9) to correct $V$ at the boundary. This is the corrector step. This process is iterated until a solution of $V$ and $P$ converges for the computational domain.

In this particular simulation, the $k-\epsilon$ [15] turbulence mode is enabled. The Reynolds number $R e=\frac{v L}{\nu}$ for the water rushing down the dam far exceeds the laminar regime of air flow.

\subsubsection{HEC-RAS Solver}

There are two options for solvers in HEC-RAS, the simplified diffusion equation versus the full momentum shallow water equation. The faster simplified diffusion equation is used initially but it produced unreasonably high water depth leading the water flow downstream. The full momentum equation 14 is computationally more expensive but does produce more realistic water depth results compared with the simplified diffusion equations.

$$
\begin{aligned}
\frac{\partial \mathbf{U}}{\partial t}+\frac{\partial \mathbf{F}_{\mathbf{x}}}{\partial x}+\frac{\partial \mathbf{F}_{\mathbf{y}}}{\partial y} & =\mathbf{S} \\
\mathbf{U} & =\left[\begin{array}{c}
h \\
h u \\
h v
\end{array}\right] \\
\mathbf{F}_{\mathbf{x}} & =\left[\begin{array}{c}
h u \\
h u u+1 / 2 g h^{2} \\
h u v
\end{array}\right] \\
\mathbf{F}_{\mathbf{y}} & =\left[\begin{array}{c}
h u \\
h u v \\
h v v+1 / 2 g h^{2}
\end{array}\right] \\
\mathbf{S} & =\left[\begin{array}{c}
0 \\
-g h \frac{\partial z}{\partial x}-c_{f} u \\
-g h \frac{\partial z}{\partial y}-c_{f} v
\end{array}\right]
\end{aligned}
$$

The bottom friction coefficient $c_{f}$ can be calculated from the Manning formula $c_{f}=$ $\frac{n^{2} g \sqrt{u^{2}+v^{2}}}{R}$ and is dependent on the Manning's coefficient $n$ [20].

\section{Results}

Both models are able to model the downstream water flow and inundation map successfully. In this section, we discuss the main results simulated from both models.

\subsection{HEC-RAS Results}

The development of the flooding pattern can be seen in Figures 9 after 4 minutes, 10 after 11 minutes, and 11 after one hour.

Table 1 shows the water flow extension per minute as a function of time. For example the table indicates 1079.2 meters of flooding occurs from minute 0 to minute 1 and from minute 1 to 2 , the length of flooding increases by 663.8 
meters. Since the flooding diverges into east and west directions, the table measures only the river's expansion in the west direction. The extension from minute 0 to 1 is measured from the base of the dam to the furthest point the water reaches along the river. The flow extension between minute 1 to minute 2 is measured from the furthest point the water reaches at minute 1 to the furthest point the water reaches at minute 2. This is repeated for the next 20 minutes as shown in the table. As expected, the water flows rapidly upon exiting the breach, gaining kinetic energy as it falls. The extension then slows as the water interacts with the terrain and flows further away from the dam, attaining a relatively constant speed of around $60 \mathrm{~m} / \mathrm{s}$ as shown in Figure 19. Overall, the water flows slower compared to OpenFOAM and has less variation in velocity.
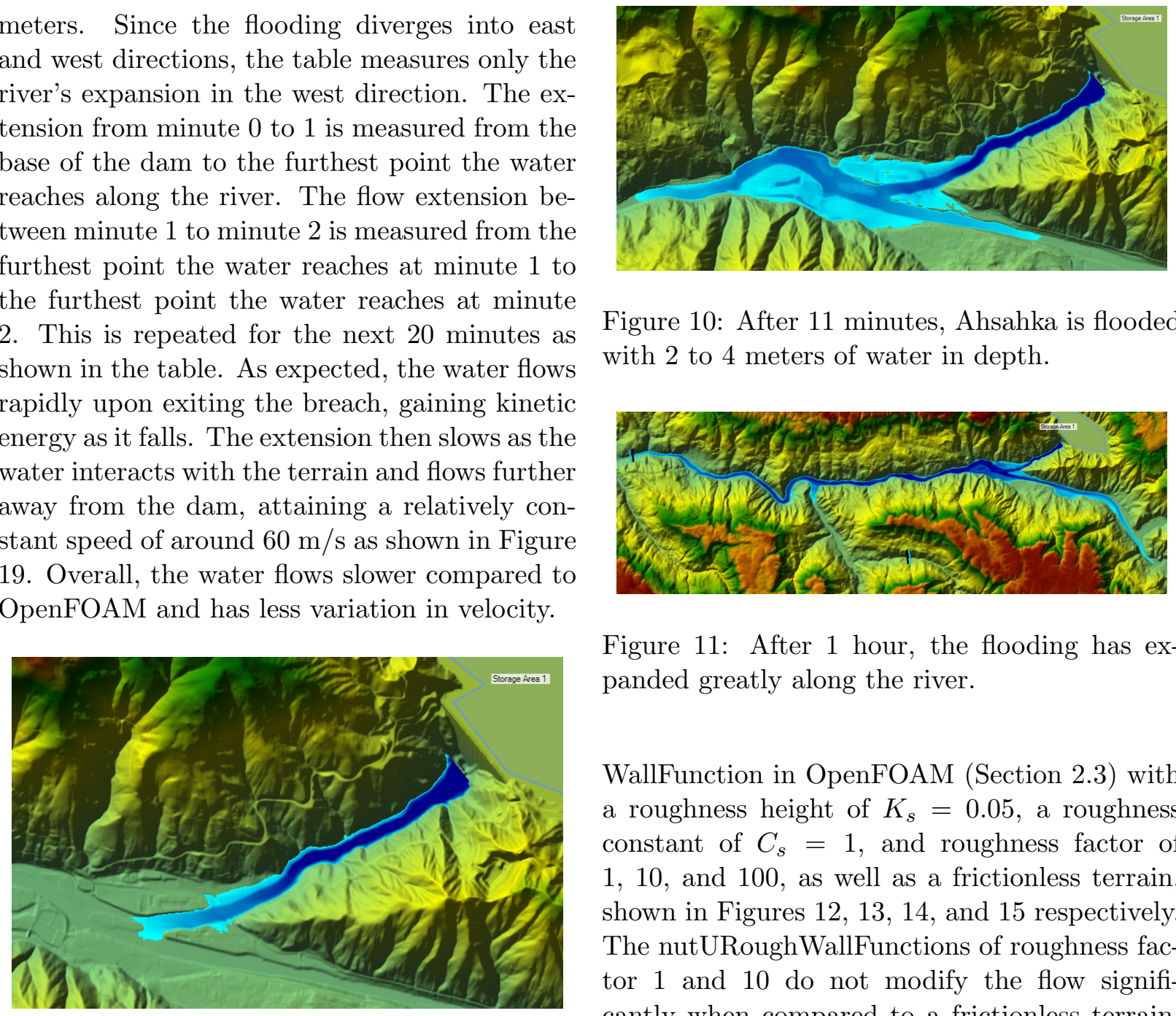

Figure 10: After 11 minutes, Ahsahka is flooded with 2 to 4 meters of water in depth.

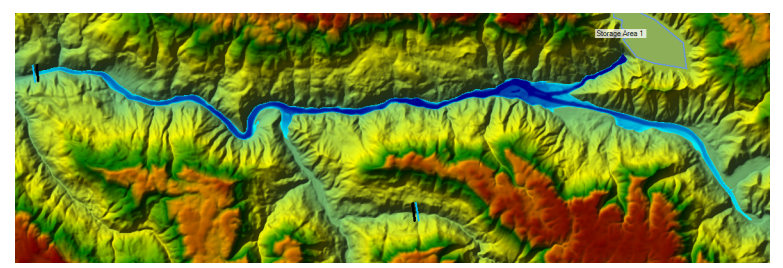

Figure 11: After 1 hour, the flooding has expanded greatly along the river.

WallFunction in OpenFOAM (Section 2.3) with a roughness height of $K_{s}=0.05$, a roughness constant of $C_{s}=1$, and roughness factor of 1,10 , and 100 , as well as a frictionless terrain, shown in Figures 12, 13, 14, and 15 respectively. The nutURoughWallFunctions of roughness factor 1 and 10 do not modify the flow significantly when compared to a frictionless terrain.

Figure 9: The water reaches Ahsahka (the small town located at the junction of the river) within 4 minutes.

\subsection{OpenFOAM Results}

In order to obtain a reasonable roughness factor for the simulation, the first 60 seconds of flow are simulated for terrains using nutURoughThe nutURoughWallFunction with a roughness factor of 100 reduces the distance traveled by 9.8\% when compared to the frictionless terrain and matches HEC-RAS closely. Within the first minute, the water travels 1106 meters in OpenFOAM and 1079 meters in HEC-RAS.

The flow is simulated for 1 hour using a nutURoughWallFunction with roughness factor 100 for the terrain. Figures 16, 17, and 18 display the flooding at 4 minutes, 11 minutes, and 


\begin{tabular}{|c|c|}
\hline Time & Water Flow Extension Per Minute \\
\hline 1 & 1079.2 \\
\hline 2 & 663.8 \\
\hline 3 & 586.9 \\
\hline 4 & 607.6 \\
\hline 5 & 372.0 \\
\hline 6 & 382.0 \\
\hline 7 & 414.4 \\
\hline 8 & 374.6 \\
\hline 9 & 395.4 \\
\hline 10 & 407.5 \\
\hline 11 & 365.4 \\
\hline 12 & 393.4 \\
\hline 13 & 358.2 \\
\hline 14 & 353.5 \\
\hline 15 & 359.9 \\
\hline 16 & 327.2 \\
\hline 17 & 375.4 \\
\hline 18 & 335.2 \\
\hline 19 & 384.2 \\
\hline 20 & 325.4 \\
\hline
\end{tabular}

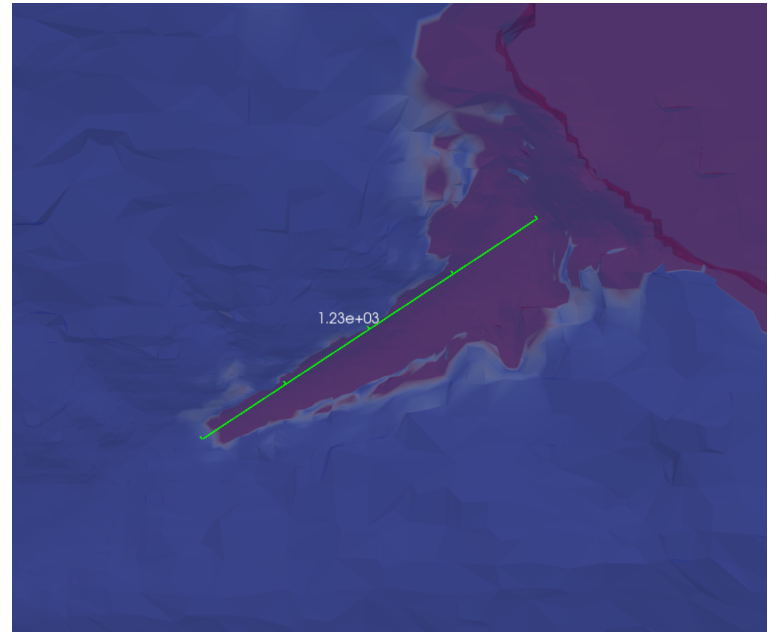

Figure 12: Length of water measured from base of dam after 60 seconds and roughness factor 1

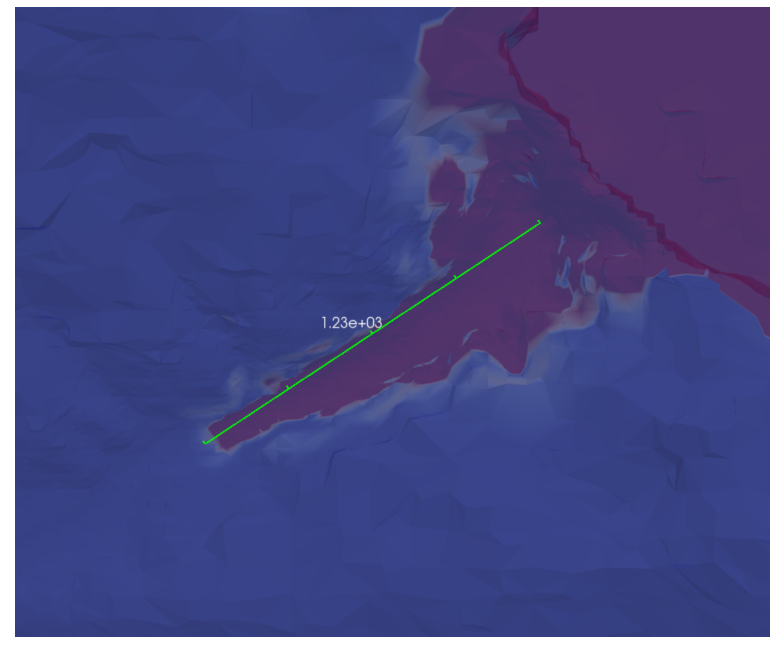

Table 1: The increase in the extension of water flow downstream (in meters) as a function of time (in minute) in HEC-RAS.

one hour respectively corresponding to the HECRAS Figures 9, 10, and 11.

Table 2 displays the extension in the flow for each minute using the same method used for Table 1. The flow extension for the first minute is measured from the base of the dam to the furthest point the water reaches after 1 minute. The data values for the next 19 minutes measure the distance from the previous furthest point of flooding to the new furthest point of flooding.

After 13 minutes, the water slows down dramatically before speeding up again. The slowing of the water coincides with the water reach-

Figure 13: Length of water measured from base of dam after 60 seconds and roughness factor 10

ing the boundary between cells with resolution $58.01 \times 52.81 \times 13.9$ meters and cells with resolution $29.01 \times 26.40 \times 6.9$ meters. It's discovered that water flow in OpenFOAM slows down when travelling from high resolution cells to low 


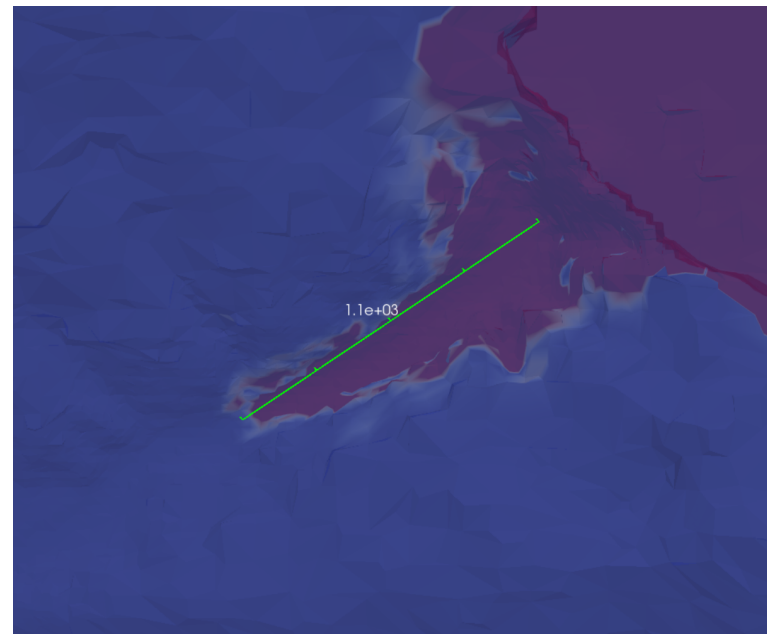

Figure 14: Length of water measured from base of dam after 60 seconds and roughness factor 100

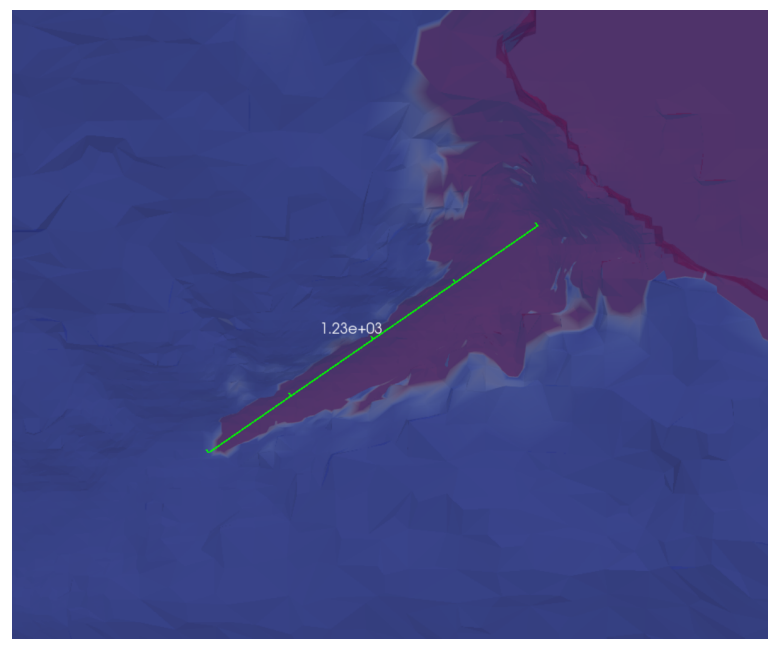

Figure 15: Length of water measured from base of dam after 60 seconds with frictionless terrain

resolution cells. The water speed then increases after entering the low resolution mesh. The acceleration of the water after crossing the boundary between high and low resolution cells can be attributed to the water's dependence on resolu- tion, with water moving faster in low resolution meshes. OpenFOAM's flow has large variations in the waterflow extension as shown in Figure 19 Overall, OpenFOAM has a faster flow as shown in Figure 20.

\begin{tabular}{|c|c|}
\hline Time & Water Flow Extension Per Minute \\
\hline 1 & 1106.8 \\
\hline 2 & 694.6 \\
\hline 3 & 948.7 \\
\hline 4 & 525.0 \\
\hline 5 & 477.6 \\
\hline 6 & 575.0 \\
\hline 7 & 421.6 \\
\hline 8 & 475.7 \\
\hline 9 & 403.9 \\
\hline 10 & 427.2 \\
\hline 11 & 412.3 \\
\hline 12 & 364.0 \\
\hline 13 & 410.0 \\
\hline 14 & 290.0 \\
\hline 15 & 210.9 \\
\hline 16 & 165.5 \\
\hline 17 & 283.9 \\
\hline 18 & 539.4 \\
\hline 19 & 550.0 \\
\hline 20 & 650.3 \\
\hline
\end{tabular}

Table 2: The increase in the extension of water flow downstream (in meters) as a function of time (in minute) in OpenFOAM.

\subsection{Resolution Dependence}

The flow of the water in HEC-RAS has negligible dependence on resolution. Changing the mesh resolution from 30 by 30 meters to 60 by 60 meters results in the water flowing 9.3 meters further during the first 60 seconds. This is 


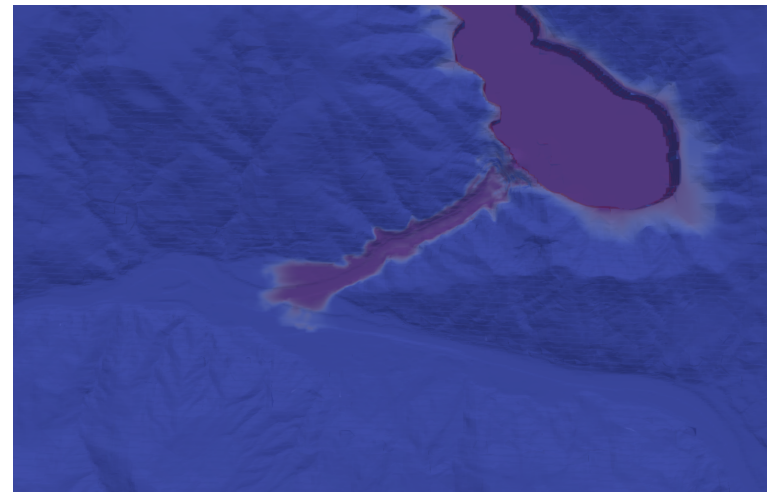

Figure 16: The water in OpenFOAM reaches Ahsahka within 4 minutes.

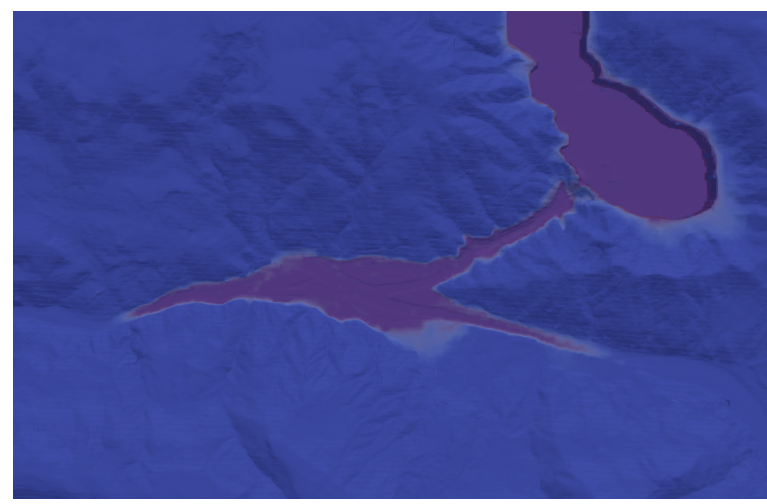

Figure 17: After 11 minutes, the flooding has completely covered Ahsahka and is slightly larger than the flooding in HEC-RAS.

only $0.9 \%$ more than the length the water travelled at 30 by 30 meter resolution. In comparison, OpenFOAM's flow is very sensitive to resolution changes. In particular, water slows down or stops at the boundary of the cells. Simulating the first 60 seconds of the dam break shows that water also tends to flow faster in a lower resolution mesh compared to a higher resolution mesh. Table 3 shows the distance the water travels in the first 60 seconds for a mesh with a low reso-

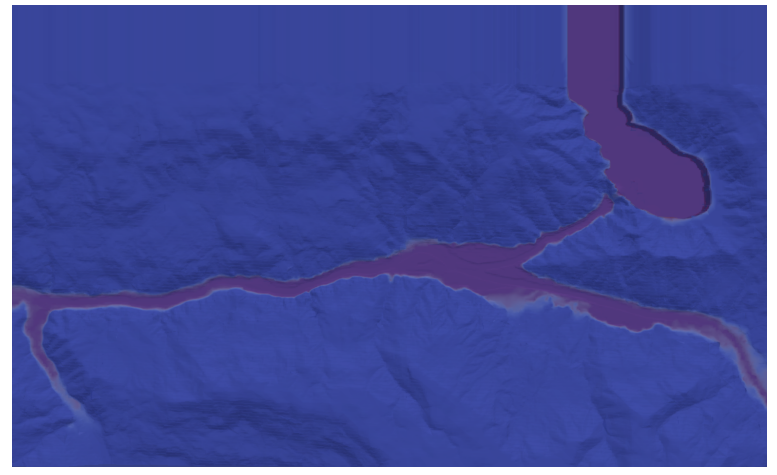

Figure 18: After 1 hour, flooding has expanded to cover the entire stretch of river meshed in OpenFOAM.

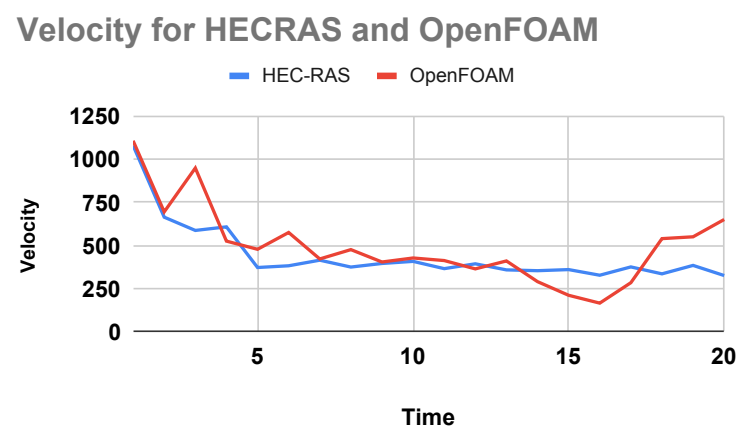

Figure 19: OpenFOAM and HEC-RAS's water flow velocity (meter/min). HEC-RAS and OpenFOAM are in good agreement with more variation in OpenFOAM due to mesh resolution difference.

lution of $67.68 \times 39.76 \times 13.9$ meters along the river compared to a mesh with a high resolution of $29.01 \times 26.40 \times 6.9$ for different roughness factors. For roughness factors of 1 and 10, the lower resolution mesh travels roughly $30 \%$ faster and for a roughness factor of 100, the low resolution mesh travels $14 \%$ faster. 
5 CONCLUSION AND FUTURE WORK

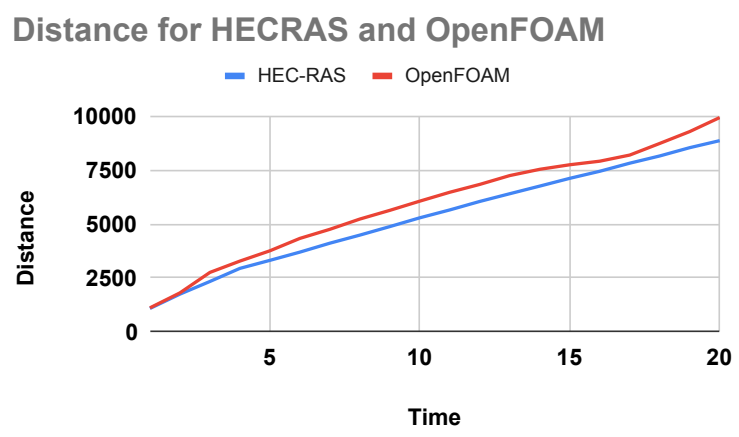

Figure 20: Distance (in meters) travelled by water in OpenFOAM is consistently greater than HEC-RAS. Water velocity OpenFOAM is slightly faster than HEC-RAS.

\begin{tabular}{|c|c|c|}
\hline Roughness Factor & Low Res & High Res \\
\hline 1 & 1620 & 1230 \\
\hline 10 & 1600 & 1230 \\
\hline 100 & 1250 & 1100 \\
\hline
\end{tabular}

Table 3: The distance the water travels (in meters) in the first 60 seconds for a low resolution mesh and a higher resolution mesh with varying roughness factors 2.3 .

\section{Conclusion and future work}

Based on a 3D digital elevation map, the potential flooding area downstream of Dworshak dam is modelled with state of the art CFD softwares, HEC-RAS and OpenFOAM. The two simulations are set up as similar to each other as possible in initial condition, boundary condition, geometry and meshing.

Despite differences in numerical schemes and the treatment of roughness boundary conditions, the overall results including flow velocity and flooding area from OpenFOAM are in good agreement with HEC-RAS. There are two no- table differences between HEC-RAS and OpenFOAM's results.

First, OpenFOAM's simulation shows significant dependences on mesh resolution: 1) The overall water flow extension and velocity increases as mesh resolution decreases (Table 3); 2) Modelled water velocity can increase or decrease when water flows through mesh regions of different resolution. Our results presented in Section 4 are based on a 30 by 30 meter resolution in OpenFOAM which shows good agreement with HEC-RAS. We suspect the resolution dependent behavior could be caused by an implementation issue in OpenFOAM.

Second, the modelled water depth in OpenFOAM significantly exceeds that modelled by HEC-RAS, evidenced by Table 4. Water depth values at 4 identical points over Ahsahka are tabled and the OpenFOAM results are much greater than the HEC-RAS results. It's not exactly clear what is causing a difference of such scale. We hypothesize that the difference is due to OpenFOAM solves the flow field with full blown 3D Navier-Stokes equations while HECRAS uses the $2 \mathrm{D}$ shallow water equations.

\begin{tabular}{|c|c|c|}
\hline Lat, Lon & HEC-RAS & OpenFOAM \\
\hline $46.505,-116.329$ & 1.82 & 53.6 \\
\hline $46.500,-116.333$ & 1.66 & 119.7 \\
\hline $46.504,-116.342$ & 2.50 & 153.5 \\
\hline $46.502,-116.322$ & 1.67 & 48.5 \\
\hline
\end{tabular}

Table 4: The depths (in meters) of flooding for specific points are shown for HEC-RAS and OpenFOAM at 11 minutes of simulation time.

Further work should look into the origin of OpenFOAM's dependence on mesh resolution and excessive water depth modelled. The dependence on mesh resolution in particular is con- 
cerning.

\section{References}

[1] Science Engineering Sustainability (2019), Science Engineering Sustainability: HEC-RAS evolution. ht tps://sciengsustainability.blogspot.com/2016 /08/some-months-ago-new-version-of-hec-ras .html Retrieved 1 October 2019.

[2] US Army Corps of Engineers (2019), https://ww w.hec.usace.army.mil/software/hec-ras/. Retrieved 28 October 2019.

[3] Jasak H., "Error Analysis and Estimation for the Finite Volume Method with applications to Fluid Flows", Direct, M

[4] H. G. Weller, G. Tabor, H. Jasak, C. Fureby, A tensorial approach to computational continuum mechanics using object-oriented techniques, COMPUTERS IN PHYSICS, VOL. 12, NO. 6, NOV/DEC 1998.

[5] Raman, A. and Liu, F. (2019), An investigation of the Brumadinho Dam Break with HEC RAS simulation, arXiv:1911.05219

[6] Lee, C. and Liu, F. (2020). Potential environmental hazard due to tailings dam failure in Minnesota iron range and safety concern of the Dams in the US. DOI: $10.31223 /$ osf.io/qbrkg.

[7] Lee, K. and Liu, F. (2021). HEC-RAS simulation of the India Dam Break and investigation of its cause.

[8] Sills, J. (2019), Brazil's Policies stuck in the mud, Science, 363, 6431

[9] Devantier, B. A. (1993), TP-144, Review of GIS Applications in Hydrologic Modeling

[10] U.S. Geological Survey, 2019, 3D Elevation Program 1-Meter Resolution Digital Elevation Model (published 20200606), accessed September 7, 2021 at URL https://www.usgs.gov/core-sciencesystems/ngp/3dep/data-tools

[11] H. K. Versteeg and W. Malalasekera, An Introduction to Computational Fluid Dynamics: The Finite
Volume Method Longman Scientific and Technical, 1995.

[12] J. H. Ferziger and M. Peric', Computational Methods for Fluid Dynamics Springer, Berlin, 1996.

[13] Owen, S. J. 1998. A survey of unstructured mesh generation technology. In Proc. 7th Inter. Mesh. Roundtable, 239-267.

[14] Community, B. O. (2018). Blender - a 3D modelling and rendering package. Stichting Blender Foundation, Amsterdam. Retrieved from http://www.blender.org

[15] Henk Kaarle Versteeg, Weeratunge Malalasekera (2007). An Introduction to Computational Fluid Dynamics: The Finite Volume Method.

[16] Folayan, Adewale Anawe, Paul Abioye, Oluwabunmi Elehinafe, F.. (2017). Selecting the Most Appropriate Model for Rheological Characterization of Synthetic Based Drilling Mud. International Journal of Applied Engineering Research. 12. 7614-7629.

[17] Messaoudi, A., Bouzit, M. Boualla, N. Physical and rheological properties of the Chorfa dam mud: dependency on solids concentration. Appl Water Sci 8, 178 (2018). https://doi.org/10.1007/s13201-0180805-8

[18] Westermaier, S. and Kowalczyk, W. (2020) Implementation of Non-Newtonian Fluid Properties for Compressible Multiphase Flows in Open- FOAM. Open Journal of Fluid Dynamics, 10, 135-150. https://doi.org/10.4236/ojfd.2020.102009

[19] Khalili-Garakani, Amirhossein Mostoufi, Navid Sadeghi, Fatemeh Hosseinzadeh, M Fatoorehchi, Hooman Sarrafzadeh, Mohammad MEHRNIA, MOHAMMAD. (2011). Comparison between different models for rheological characterization of activated sludge. Iranian Journal of Environmental Health Science Engineering. 8. 255-264.

[20] US Army Corps of Engineers, (2014), Using HECRAS for Dam Break Studies. 
[21] George J. Arcement, Jr., Verne R. Schneider. (1989). Guide for Selecting Manning's Roughness Coefficients for Natural Channels and Flood Plains. https://doi.org/10.3133/wsp2339

[22] Casson, N. (1959) A Flow Equation for Pigment-Oil Suspensions of the Printing Ink Type. In: Mill, C.C., Ed., Rheology of Disperse Systems, Pergamon Press, Oxford, 84-104.

[23] Swati Mukhopadhyay, Prativa Ranjan De, Krishnendu Bhattacharyya, G.C. Layek, Casson fluid flow over an unsteady stretching surface, Ain Shams Engineering Journal, Volume 4, Issue 4, 2013, Pages 933-938, ISSN 2090-4479, https://doi.org/10.1016/j.asej.2013.04.004. 